\title{
Characterisation of the boson peak from the glass into the liquid
}

\author{
Andrew J. Farrell, Mario González-Jiménez, Nikita V. Tukachev, David A. Turton, Ben A. Russell, Sarah \\ Guinane, Hans M. Senn, Klaas Wynne* \\ School of Chemistry, University of Glasgow, UK
}

\begin{abstract}
Phenomena ranging from vitrification to crystal nucleation are governed by locally ordered structures, in otherwise disordered phases, that can either inhibit or favour the growth of macroscopic order. However, such structures are ephemeral, do not typically have distinct spectral features, and are therefore critically important but largely unobservable by current methods. Illuminating these structures therefore presents the single greatest challenge in physical chemistry. The boson peak is characteristic of glasses and represents the locally ordered structures inhibiting crystallisation but is typically obscured by other spectral contributions. Here we show that depolarised Raman scattering-obtained using femtosecond optical Kerr-effect spectroscopy -in liquids consisting of highly symmetric molecules can be used to isolate the boson peak thereby allowing detailed characterisation of the intermolecular potential-energy landscape for the first time.
\end{abstract}

The expected behaviour of a liquid when cooled is to freeze at the melting temperature, where the molecules arrange themselves in an ordered crystalline state. However, many liquids can be supercooled where crystallisation is postponed. On further cooling, the supercooled liquid becomes increasingly viscous and at the glass-transition temperature vitrifies into an amorphous solid called a glass. The liquid-glass transition is one of the most difficult and well-studied problems of condensed matter physics. ${ }^{\mathrm{I}-5}$

A common and quintessential feature of glasses is the observation of the so-called boson peak typically seen in spontaneous Raman scattering spectroscopy and inelastic neutron scattering at a frequency around I THz. The boson peak represents a peak in the vibrational density of states, $g(\omega)$, normalised by the frequency squared, $g(\omega) / \omega^{2}{ }^{6}$ This corresponds to an excess in the density of states over that expected from phonons in a perfect Debye crystal. Thus, the boson peak signals a crossover in the vibrational density of states from phonon like $\omega^{2}$ scaling at low frequency to $\omega^{n}(n>2)$ scaling at high frequency. This gives rise to anomalous behaviour of the lowtemperature heat capacity $C_{p}$ and a peak in $C_{p} / T^{3}$ at a few Ios of $\mathrm{K}$. The boson peak has a predominantly transverse character $^{7-9}$ and is strongest in, e.g., depolarised Raman scattering. The boson peak typically becomes visible when a (supercooled) liquid is vitrified. Thus, the position, amplitude, and shape of the boson peak could provide crucial information on the physics of supercooling and vitrification. The origin of the boson peak is still the subject of much scrutiny to date, with many proposed mechanisms to explain how it emerges. Some believe it to be caused by random spatial fluctuations of elastic constants brought about by disorder. ${ }^{\mathrm{IO}-\mathrm{I} 2}$ It has also been argued to be the remnant of a van Hove singularity of the corresponding crystal, that has been washed out by amorphous structure. ${ }^{13,14}$ Other explanations include the coupling of acoustic phonon modes with quasi-localised soft modes arising from defects, ${ }^{15,16}$ or the weak interaction between quasi-localised harmonic modes producing anharmonicity. ${ }^{17}$ In supercooled liquids, Tanaka argues that crystal nucleation is inhibited by locally favoured structures that geometrically inhibit the formation of the thermodynamically most stable state and give rise to the emergence of the boson peak. ${ }^{{ }^{8}}$ Thus, the spectral features of the boson peak could provide crucial insight into the nature and abundance of these locally favoured structures.

However, in most liquids (including those that vitrify) it is difficult to distinguish the various processes that contribute to the vibrational spectrum in the low-terahertz range. In this range in liquids, one can distinguish spectral contributions from $^{19,20} \alpha$-relaxations due to orientational and translational diffusion, an intermolecular oscillatory mode (sometimes referred to as "cage rattling"), librational motion, and finally low-frequency vibrational modes. The $\alpha$-relaxation processes freeze out on vitrification while vibrational contributions are sometimes narrow and relatively easy to distinguish. That leaves the cage-rattling intermolecular mode and librations in addition to possible contributions from a boson peak, all of which peak around I THz, making an unobscured observation of the boson peak difficult to impossible.

Thus, there are numerous reports of observations of boson peaks in the literature, however, many of these are potentially questionable. In depolarised Raman scattering, the intensity of both the orientational-relaxation and librational bands scale with the anisotropic part of the polarisability tensor and therefore presence of one implies the other. Thus reports of boson peaks observed in the Raman spectra of vitrified $\mathrm{B}_{2} \mathrm{O}_{3},{ }^{21,22}$ $\mathrm{As}_{2} \mathrm{~S}_{3},{ }^{23} \mathrm{Ca}_{2} \mathrm{~K}_{3}\left(\mathrm{NO}_{3}\right)_{7},{ }^{22}$ propylene glycol, ${ }^{24} \mathrm{~m}$-tricresyl phosphate, ${ }^{2 \mathrm{I}}$ and salol ${ }^{25}$ are suspect as these show orientational-relaxation in the liquid implying the presence of a librational band rather than a boson peak. Interpretation of the Raman spectrum of glycerol ${ }^{21,26}$ also suffers in this respect, however the validity of the boson peak assignment has been strengthened with results from neutron scattering experiments. ${ }^{27}$

Raman and inelastic neutron scattering spectra of $\mathrm{Y}_{2} \mathrm{O}_{3-}$ $\mathrm{Al}_{2} \mathrm{O}_{3}{ }^{28} \quad \mathrm{Na}_{0.5} \mathrm{Li}_{0.5} \mathrm{PO}_{3}{ }^{29} \quad \mathrm{ZnCl}_{2}$ and $\mathrm{ZnBr}_{2},{ }^{30}$ ortho-terphenyl, ${ }^{31,32}$ polyisoprene, ${ }^{1232}$ polystyrene, ${ }^{32}$ and other polymers ${ }^{33}$ are complicated by vibrational or phonon bands making it difficult to unambiguously identify a boson peak in the vitrified liquids. The Raman spectra of glassy sulfur ${ }^{34}\left(\mathrm{~S}_{8}\right)$ and selenium ${ }^{35}$ show a complex band shape between o and Ioo$\mathrm{I} 50 \mathrm{~cm}^{-1}$, the high frequency region of which has been correctly assigned to librations. However, bumps at circa $20 \mathrm{~cm}^{-1}$ ( $\sim .6 \mathrm{THz})$ are assigned to (and could well be) boson peaks but reliable analysis of their (temperature dependent) shape and amplitude is not possible. This leaves the peak observed at $\sim 50 \mathrm{~cm}^{-1}$ (I.6 THz) in glassy $\mathrm{SiO}_{2}, \mathrm{GeO}_{2}$, etc., which were assigned to a boson peak. ${ }^{36-41}$ Although this peak could be due to librations or torsional vibrations, as the local $\mathrm{SiO}_{4}$ structure is likely to be close to tetrahedral (where the anisotropic part of the polarisability tensor would vanish and which would make the librational band invisible in depolarised Raman scattering), this might well be an actual boson peak. In summary, although observation of the boson peak in vitrified liquids has been reported many times over, some of these reports are unreliable while in the remaining cases it is difficult to determine the 
shape and amplitude for detailed analysis. However, the example of glassy $\mathrm{SiO}_{2}$ shows the way forward: symmetry can be used to simplify depolarised Raman spectra.

Here, we study glass-forming titanium alkoxide liquids in which the molecules have a high degree of symmetry, thereby eliminating the signals from orientational diffusion and librations. On vitrification, diffusive translations freeze out, leaving only the intermolecular mode, whose signal in depolarised Raman spectroscopy scales with intermolecular collision strength. This intermolecular mode corresponds to rattling of molecules in the cage formed by their neighbours. ${ }^{20,42,43}$ Thus, in a model in which the supercooled liquid consists of liquidlike regions and locally favoured structures (whose size and/or preponderance increases on lowering temperature, see Fig. I), then both should show such cage-rattling intermolecular modes. We will argue that these are the much-vaunted boson peak. In order to achieve optimum signal-to-noise in the (sub)terahertz region, we use femtosecond optical Kerr-effect (OKE) spectroscopy, which measures the depolarised Raman spectrum using a time-domain technique. ${ }^{44^{-46}}$ We show that the simplified spectra of such carefully selected materials can reveal the temperature-dependent behaviour of an unambiguous boson peak not only in the vitreous state, but well into the liquid state at temperatures as much as $190 \mathrm{~K}$ above $T_{g}$. Similar experiments on the well-known glass former toluene show that it has an identically behaving boson peak hidden under many other contributions to the spectrum.

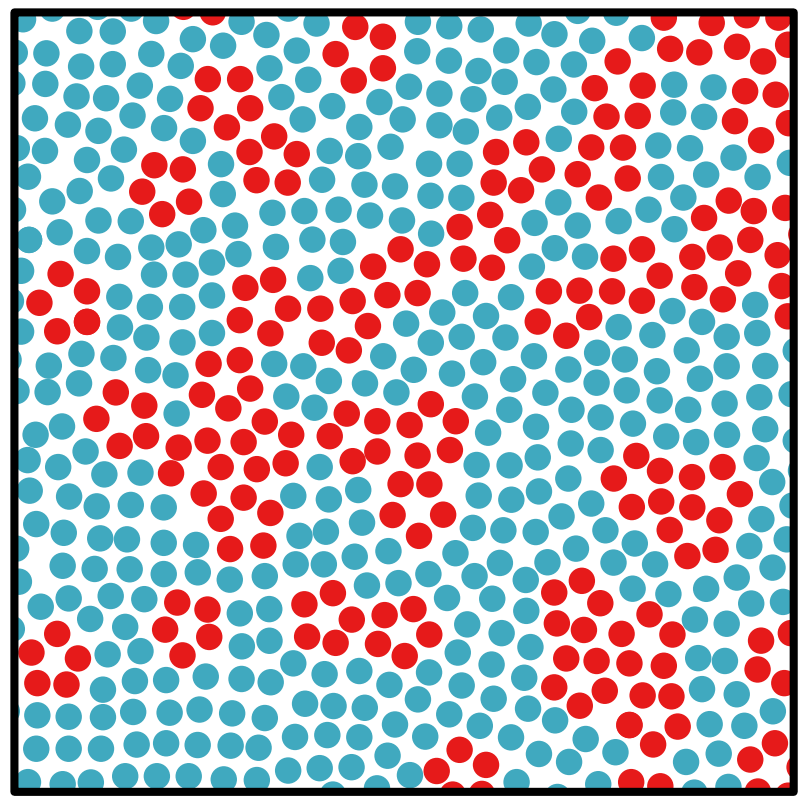

Fig. 1. In a supercooled liquid, locally favoured structures form in a sea of normal liquid structures. It has been assumed here that locally favoured structures (red) have five-fold symmetry and therefore cannot tile space whereas the normal liquid (blue) lacks structure. Adapted from Ref. ${ }^{47}$.

\section{Results}

The hidden boson peak in toluene. The glass-forming liquid toluene $\left(T_{g}=\mathrm{I} 2 \mathrm{OK}\right)$ will be used to illustrate the difficulty in determining the presence of a boson peak. The boson peak is directly related to the extra heat capacity of the glass over the crystal. In toluene, the heat capacity $C_{p}$ has been measured for stable $^{48}$ (liquid, crystal) and metastable ${ }^{49}$ (super cooled, glass) sequences. As can be seen in Fig. 2, vitrified toluene has $\sim \mathrm{IO} \%$ extra heat capacity over the crystal, which peaks at a temperature $T_{\text {boson }}=\sim 20 \mathrm{~K}$. This must be due to an "extra" vibrational density of states, that is, a boson with a frequency that would be estimated as $k_{B} T_{\text {boson }} / h$ or $\sim 0.4 \mathrm{THz}$ in the case of toluene.

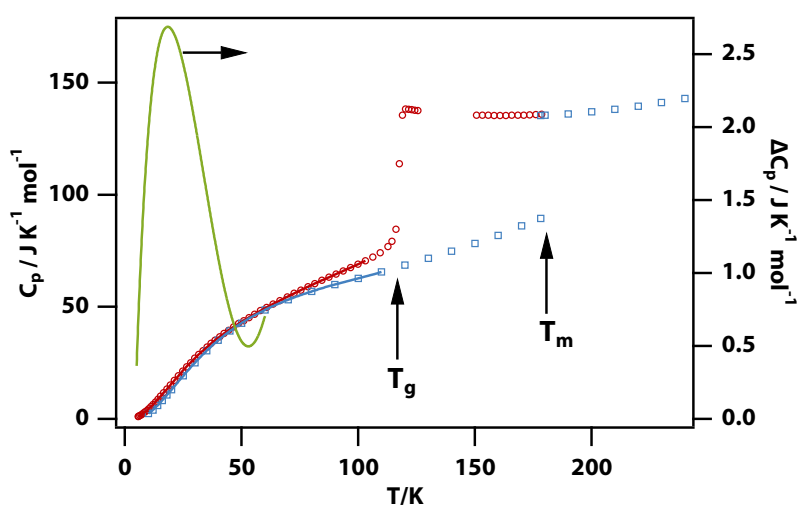

Fig. 2. Molar heat capacities of metastable and stable sequences of toluene. Shown are supercooled and vitrified toluene (red circles), ${ }^{49}$ liquid and crystalline toluene (blue squares) ${ }^{48}$ fits to $8^{\text {th }}$ order polynomials (red and blue solid lines), and the difference between these fits (green line). The difference heat capacity peaks at $20 \mathrm{~K}$ corresponding to $0.42 \mathrm{THz}$.

The temperature-dependent terahertz spectra of toluene taken using OKE spectroscopy are shown in Fig. 3(inset). Standard processing of the time-domain OKE data results in a reduced depolarised Raman spectrum (often referred to as the susceptibility representation of the anisotropic Raman spectrum) in which the low-frequency $(<\mathrm{I} \mathrm{THz})$ part of the spectrum is easily visualised. The reduced spectrum can be converted to a spontaneous Raman scattering spectrum by multiplying with a Bose occupation factor (see equation $\mathrm{S}_{5}$ in Supplementary Note I). This converted spontaneous Raman spectrum is shown in Fig. 3 and is consistent with published Raman spectra of toluene..$^{50,5 \mathrm{I}}$ In liquid and supercooled toluene, the spontaneous Raman spectrum is dominated by so-called elastic scattering. The elastic peak vanishes only when toluene is fully vitrified, to reveal a peak at $2.5 \mathrm{THz}$ and a weaker shoulder at $0.5 \mathrm{THz}$. In similar temperature-dependent spontaneous Raman and inelastic neutron scattering spectra, the higher frequency peak is often identified as the boson peak but is in fact due to molecular librations..$^{52}$

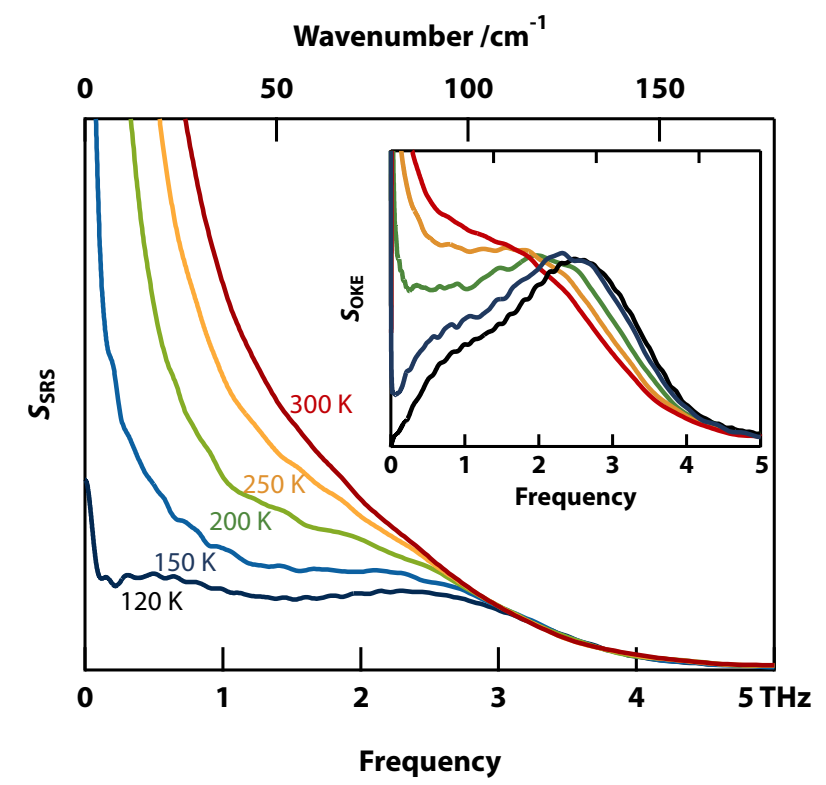

Fig. 3. Temperature dependent terahertz spectra of toluene Shown are the OKE spectrum (inset) and the spontaneous anisotropic 
Raman spectrum (SRS, main panel) derived from it on the bottom. In practice, experimental spontaneous Raman scattering has poor signal-to-noise below circa $1 \mathrm{THz}$.

The 0.5-THz shoulder might well be the actual boson peak (the frequency is right based on excess heat capacity measurements, see above) but its shape cannot be determined with great accuracy, and it cannot be ruled in or out that it exists in the supercooled liquid as well. Note that the OKE spectra do not show a huge elastic peak in the liquid phase but instead have (eminently analysable, see for example Ref. ${ }^{19}$ ) sub-terahertz bands due to diffusive processes such as orientationaland translational-relaxation. Thus, the case of toluene illustrates the complications in determining the presence and characteristics of boson peaks.

Spectral simplification in titanium alkoxides. In order to simplify the spectra, we carried out an extensive search for molecular liquids consisting of molecules with high symmetry (tetrahedral, octahedral, or icosahedral), including ionic liquids with high symmetry ions and eutectic mixtures. Unfortunately, liquids of highly symmetric molecules or ions have a propensity to crystallise rather than vitrify. However, some of the liquids formed by titanium(IV) alkoxides are found to be glass formers (see Fig. 4). Titanium tetraethoxide, tetrapropoxide, and tetraisopropoxide were found to crystallise readily on cooling. However, titanium tetrabutoxide $(\mathrm{TiBuO})$ and tetra-2ethylhexyloxide could be vitrified with ease. TiBuO has a glass transition at $T_{g}=\mathrm{I} 62.5 \mathrm{~K}$ as measured using DSC (see Supplementary Figure I). We have not been able to crystallise TiBuO and therefore the formal melting temperature is unknown. Analysis of measurements of the viscosity at relatively high temperatures compared to the glass transition temperature, suggests that $\mathrm{TiBuO}$ is a weakly fragile glass former (see Supplementary Figure 2).

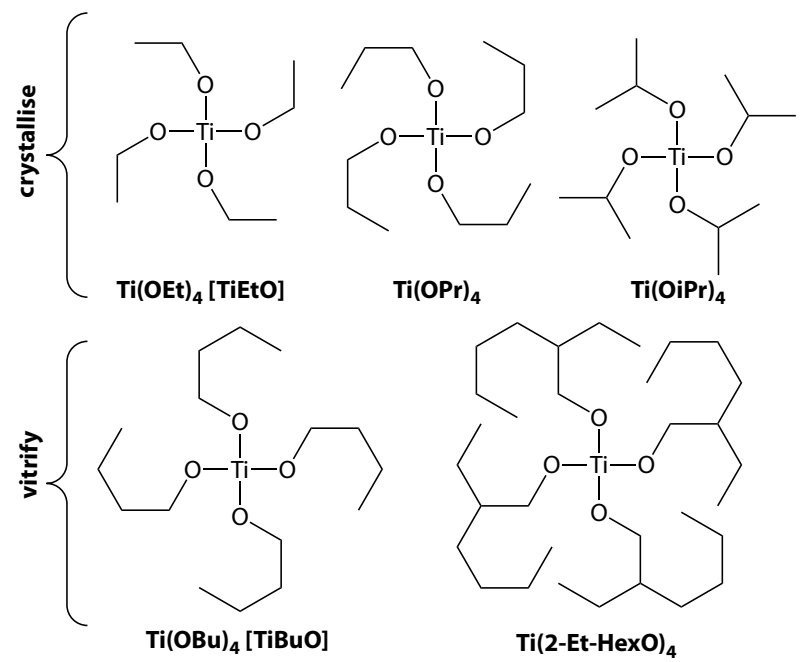

Fig. 4. Titanium alkoxides investigated. The short chain alkoxides (top) tend to crystallise while the longer chain ones (bottom) vitrify.

$\mathrm{TiBuO}$ is known not to be monomeric. The crystals of titanium tetramethoxide and tetraethoxide are known to be tetrameric, with the titanium atom being octahedrally coordinated.53 In the liquid phase, the titanium atoms in $\mathrm{TiBuO}$ are thought to be 5 -fold coordinated (but see below) in what are most probably trimers. ${ }^{54-56}$ The most probable 5-fold $\left(\mathrm{D}_{3 \mathrm{~h}}\right)$ coordination of titanium in liquid $\mathrm{TiBuO}$ might give rise to a (small) anisotropic component in the molecular polarisability. The known rapid terminal-bridging alkoxide exchange ${ }^{57}$ will be used below to demonstrate that the low-frequency OKE spectrum is predominantly collision-induced.

Fig. 5 shows the OKE spectra of TiBuO over temperatures ranging from the glassy state $(80 \mathrm{~K})$, through the glass transition temperature $(\mathrm{I} 62.5 \mathrm{~K})$ into the supercooled regime, to room temperature and above $(350 \mathrm{~K})$. The spectra show several bands at frequencies $4 \mathrm{THz}$ and higher whose amplitudes are essentially independent of temperature. These are almost certainly due to intramolecular vibrations.

Vibrational normal mode calculations were carried out and used to calculate the depolarised Raman spectrum converted into an OKE spectrum (see Supplementary Note I). As can be seen in the calculated OKE spectrum in Supplementary Figure 3 , there are modes with moderate OKE intensity throughout the low terahertz frequency range. The four lowest frequency modes are butoxide librations. Based on our previous OKE studies on alkanes, ${ }^{19}$ these librations are expected to be weak in the OKE spectrum.

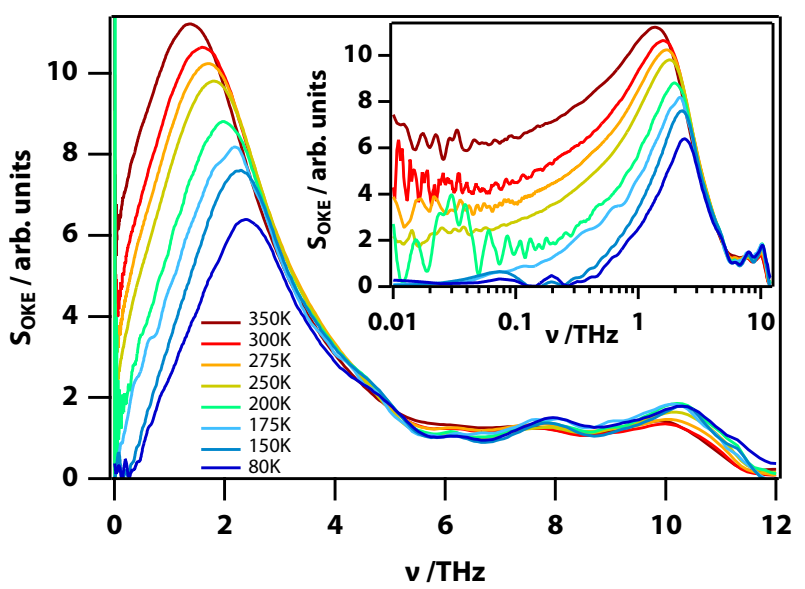

Fig. 5. OKE spectra of titanium butoxide as a function of temperature. Spectra taken at a range of temperatures from 80 to $350 \mathrm{~K}$ and shown on a linear frequency axis (main panel) and a logarithmic frequency axis (inset). The band at circa $2 \mathrm{THz}$ has a strongly temperature-dependent amplitude demonstrating it is collision induced.

The dominant feature in the spectrum in Fig. 5 is a band peaking between I and $3 \mathrm{THz}$, whose position and amplitude is strongly temperature-dependent. On a logarithmic frequency axis, it is clear that there is an additional diffusive contribution below I THz. This diffusive process shifts to lower frequency on lowering the temperature and is invisible below the glass transition temperature. Similar experiments (although much less detailed) were carried out on titanium tetra2-ethylhexyloxide (see Fig. 6), which shows the same effect. 


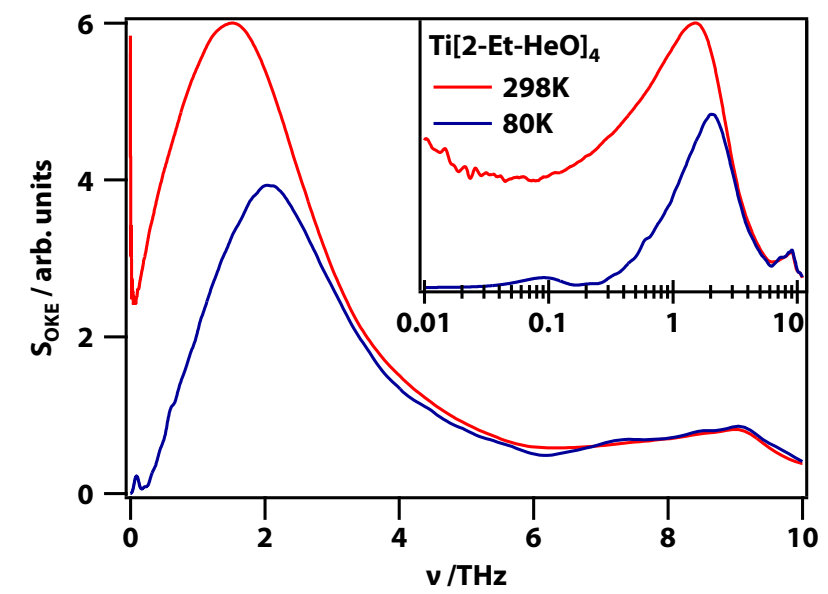

Fig. 6. OKE spectra of titanium tetra-2-ethylhexyloxide. Spectra taken at $298 \mathrm{~K}$ (red) and $80 \mathrm{~K}$ (blue), and are shown with a linear frequency axis (main) and logarithmic (inset)

Although the symmetry around the titanium atom is not tetrahedral or octahedral, the lower symmetry 5 -fold coordination of titanium in liquid $\mathrm{TiBuO}$ could potentially give rise to a librational band in addition to the butoxide librations. To rule out such librational contributions to the OKE spectra, the symmetry was lowered more severely on purpose by making 50:50 mixtures of $\mathrm{TiBuO}$ and titanium tetraethoxide (TiEtO). As the terminal and bridging ligands are known to exchange rapidly, ${ }^{57}$ this will lead to the formation of mixtures of Ti $(\mathrm{OEt})_{\mathrm{n}}(\mathrm{OBu})_{4-\mathrm{n}}$ most of which lack symmetry. The OKE spectra of TiBuO, TiEtO, and their 50:50 mixture at room temperature are shown in Fig. 7. The 50:50 mixture does not show an increased amplitude of the 1-3 THz main band but simply a band that is exactly in between that of TiBuO and TiEtO. This confirms that this band is indeed predominantly of collision-induced character, and we therefore assign this band as the intermolecular mode band. The lack of any band of librational origin implies that the diffusive process is due to translational $\alpha$-relaxation and there is no orientational $\alpha$-relaxation band visible due to symmetry.

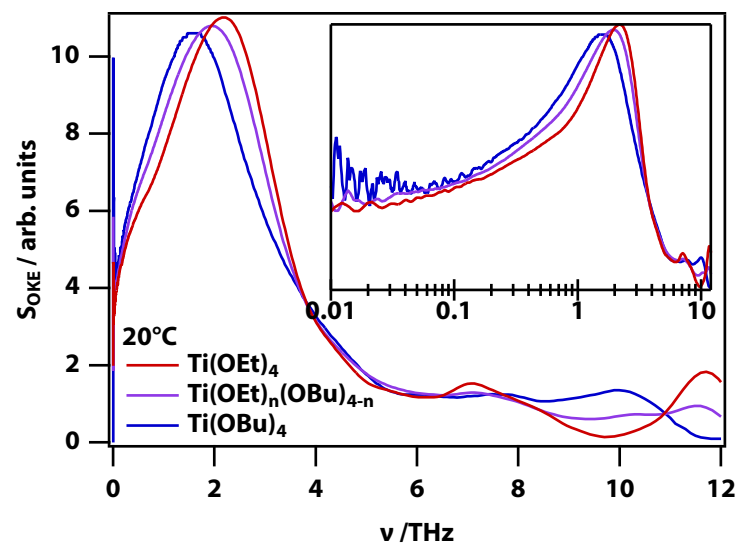

Fig. 7. OKE spectra of titanium butoxide and ethoxide and their 50:50 mixture at $20^{\circ} \mathrm{C}$. The spectrum of the 50:50 mixture is exactly in between that of the pure liquids despite consisting of mostly asymmetric $\mathrm{Ti}(\mathrm{OEt})_{n}(\mathrm{OBu})_{4-n}$ molecules.

The (temperature-dependent) OKE spectra of TiBuO can be fitted with an inertial Cole-Cole function for the translational-relaxation and Brownian-oscillator functions for the intermolecular mode and intramolecular vibrations. In the case of TiEtO and its mixture with TiBuO, the intermolecular mode could only be fitted satisfactorily with a Gaussian function, consistent with the presence of a mixture of Ti $(\mathrm{OEt})_{\mathrm{n}}(\mathrm{OBu})_{4-\mathrm{n}}$ species (see Methods for details on fit functions, and Supplementary Figures 4, 5, and 6 for the fits and Supplementary Table 2 for the fit parameters).

The relevant fit parameters for the temperature-dependent OKE spectra of TiBuO are shown in Fig. 8, Supplementary Figure 7, and Supplementary Table I. The amplitude of the weak shoulder at circa $4 \mathrm{THz}$ remains constant, consistent with an intramolecular vibrational mode. The amplitude of the intermolecular mode band increases dramatically with increasing temperature as expected for a collision-induced band. The frequency of this band decreases with increasing temperature. Below the glass transition temperature, this intermolecular mode band is the only band of intermolecular origin that remains visible (as $\alpha$-relaxations have frozen out) and can therefore be assigned as the boson peak.

The intensity of collision-induced Raman bands can be estimated using a simple model based on the molecular polarisability, the intermolecular potential, and the temperature-dependent collision energy as described in Supplementary Note 2. The resultant equation for the amplitude (equation Si6) was used to fit the amplitude of the boson peak as shown in Fig. 8. This again confirms that the boson peak is a collision induced band. There is no obvious a priori model to describe the frequency of the boson peak. Supplementary Note 3 describes a possible temperature dependence of the boson-peak frequency based on a simple Morse intermolecular potential model of the supercooled liquid. The resultant equation for the oscillator frequency (equation $\mathrm{S}_{20}$ ) was used to fit the boson-peak frequency as shown in Fig. 8.
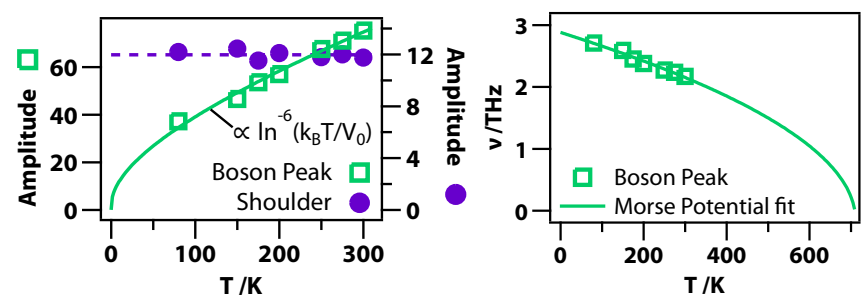

Fig. 8. Amplitude and frequency of the boson peak as a function of temperature. (left) Shown are the amplitude of the boson peak and the shoulder (an intramolecular mode). While the boson peak amplitude changes, the amplitude of the intramolecular mode is unchanged with temperature as expected. The boson-peak amplitude data are fit to equation S16 with amplitude 59 \pm 22 and $V_{0} \times 10^{17}=$ $6.3 \pm 3.9 \mathrm{~J}$. (right) Shown are the boson-peak frequency data, fit to equation $\mathrm{S}_{20}$ with $\mathrm{D}_{e} / \mathrm{h} / \mathrm{THz}=14.8 \pm 0.7$ and $\mathrm{v}_{0} / \mathrm{THz}=3.04 \pm 0.05$.

Re-evaluating the spectra of toluene. The data obtained in $\mathrm{TiBuO}$ and its analogues - due to their relatively high degree of symmetry and therefore simplified low-terahertz spectrum-show that the boson peak can be equated to the intermolecular mode. This result suggests a re-evaluation of the temperature-dependent OKE spectra of toluene discussed above.

The toluene data shown in Fig. 3 were analysed using a model we have shown to be able to fit a wide range of temperature-dependent OKE spectra of alkanes and cycloalkanes. It involves separate fit functions for (from low frequency to high) orientational and translational $\alpha$-relaxation, the intermolecular mode, and a libration (see Supplementary Figure 8 and Supplementary Table 3). It is therefore an obvious extension of the model used to fit the TiBuO data (inertial Cole-Cole for 
translational-relaxation and Brownian oscillator for the intermolecular mode) with two extra functions (Debye and Gaussian functions) to model the orientational-relaxation and libration, which are visible in toluene due to its lower symmetry but invisible in TiBuO. Satisfactory fits can be obtained throughout the glass, supercooled, and normal liquid ranges. At $\mathrm{I} 2 \mathrm{O} \mathrm{K}$, in the glass, the $\alpha$-relaxation processes have frozen out and the OKE spectrum is fitted using a librational band at high and a intermolecular mode band at low frequency. For increasing temperatures, the relaxational bands creep back in towards higher frequency, obscuring the intermolecular mode. However, a plot of the amplitude and frequency of the intermolecular mode as a function of temperature (Supplementary Figure 9) shows the exact same behaviour as in TiBuO. Hence, in toluene the intermolecular mode band can also be identified as the boson peak.

\section{Discussion}

In crystals, phonons determine the elastic properties of the solid. Glasses are amorphous solids, in which phonon-like excitations persist, giving rise to the boson peak. Raman scattering (and therefore OKE) in crystals is strictly sensitive only to modes at the zone centre corresponding to infinite wavelength phonons. The intermolecular mode gives rise to a collision-induced band that is observed in OKE experiments in many liquids and glasses, from noble-gas liquids ${ }^{5}$ through simple alkanes $^{19}$ and ionic liquids consisting of symmetric ions, ${ }^{44}$ to toluene and $\mathrm{TiBuO}$ as shown here. Its collision-induced nature implies that it is sensitive to phonon-like excitations at the Brillouin zone edge and therefore senses motions on the scale of a couple of molecules. In most cases, the intermolecular mode band is hidden but, as we have shown here, can be revealed by using molecules with a high degree of symmetry.

TiBuO does not have the high octahedral symmetry required to simplify its OKE spectrum but is likely trimeric, with most likely 5-fold (D3h) coordination of titanium. ${ }^{54-56}$ Therefore, the question arises why the OKE spectrum of TiBuO lacks the orientational-relaxation and librational bands. Quantum chemistry calculations were carried out to establish which trimeric conformers were most likely to be present in solution (see Methods and Supplementary Note 4 for details). Out of a large range of trial structures, five conformers were found to be stable (see Fig. 9; relative energies are given in Supplementary Table 4). For structures I-III, the coordination number of each titanium atom is six, while the other structures have one or two titanium atoms with a coordination of five. None of the $\mathrm{TiO}_{5}$ and $\mathrm{TiO}_{6}$ cores have perfectly octahedral or $\mathrm{D}_{3 \mathrm{~h}}$ symmetry but are notably distorted. The calculations show that the polarisability is evenly distributed over the atoms in each of the structures (Supplementary Table 5). The structures themselves have a very small anisotropic but large isotropic polarisability (Supplementary Table 6) thereby suppressing OKE contributions due to orientational relaxation and librations while enhancing collision-induced contributions. Considering the known rapid exchange of terminal and bridging alkoxides down to at least $\mathrm{I} 83 \mathrm{~K}, 57$ the conformers in Fig. 9 are to be regarded as repeating motifs in the liquid and (at low temperature) essentially a network glass. This implies that $\mathrm{TiBuO}$ is somewhat comparable in structure to vitreous $\mathrm{SiO}_{2}$.

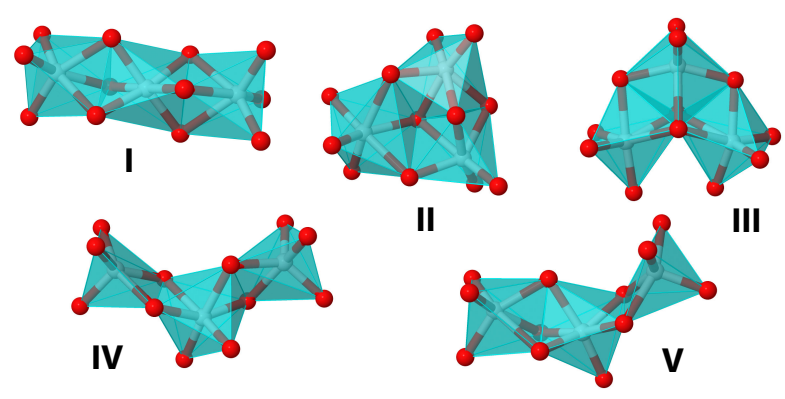

Fig. 9. Molecular models of the titanium butoxide trimeric clusters in the liquid. The number of doubly bridging $\left(\mu_{2}-O\right)$ and triply bridging alkoxide groups $\left(\mu_{3}-O\right)$ for the different clusters is I $\left(\mu_{2}-O\right.$ : 6), II( $\left.\mu_{2}-O: 4, \mu_{3}-O: 1\right)$, III( $\left.\mu_{2}-O: 2, \mu_{3}-O: 2\right), I V\left(\mu_{2}-O: 4\right), V\left(\mu_{2}-O: 5\right)$.

It is for these reasons that the $\mathrm{OKE}$ spectrum of $\mathrm{TiBuO}$ is of such a simple form, only showing bands in the low terahertz region due to translational relaxation and the intermolecular mode. The band due to the intermolecular mode remains on vitrification, demonstrating that it is the boson peak. Importantly, this band persist in the liquid state, showing that the elastic properties of the glass persist in the liquid ${ }^{59}$ albeit only on short timescales before diffusion and flow set in. The same applies to toluene, although in that case the boson peak is hidden by orientational-relaxation and librations. Our results for the boson peak in toluene correspond well with a study of toluene using probe molecules and nuclear inelastic x-ray scattering ${ }^{52}$ measuring collective motions with a correlation length of more than $\sim 20 \AA$. These reveal a boson peak with a maximum at I.I THz ( $4 \mathrm{meV}$ at $22 \mathrm{~K}$ ), closely matching the I.O THz peak of the intermolecular mode measured here at $\mathrm{I} 2 \mathrm{O} \mathrm{K}$.

The excellent quality of the OKE spectra allows a far more detailed analysis of the behaviour of the boson peak as a function of temperature. In both $\mathrm{TiBuO}$ and toluene, the boson peak shifts to higher frequencies on cooling, as predicted by theory. ${ }^{6}$ The amplitude of the boson peak decreases on lowering the temperature. However, the OKE spectrum $S^{O K E}(\omega)$ is related to the vibrational density of states $g(\omega)$ through $S^{O K E}(\omega)=$ $g(\omega) C(\omega) / \omega$, where $C(\omega)$ is the Raman coupling coefficient (see Supplementary Note I). The latter is often assumed to be proportional to $\omega$ but that is very much not true here as this is a collision-induced band whose Raman coupling coefficient strongly depends on temperature. Therefore, unfortunately, these data cannot be used directly to make comparisons with theoretical predictions of changes in the magnitude of the vibrational density of states of the boson peak as the glass transition is approached.

The boson-peak position and shape is a reflection of the potential-energy landscape ${ }^{60}$ of the (supercooled) liquid and glass. One might have expected the observation of two distinguishable intermolecular cage-rattling mode bands or a Gaussian distribution of intermolecular modes originating from liquid-like structures and locally favoured structures. ${ }^{18}$ In the case of the titanium alkoxides, it could be argued that the trimeric clusters are effectively locally favoured structures that inhibit (in the case of $\mathrm{TiBuO}$ ) or impede (in the case of TiEtO) the formation of the tetrameric crystal. However, the boson peak is well modelled by a single Brownian oscillator throughout the entire temperature range studied, with great confidence in neat $\mathrm{TiBuO}$ and less confidence in toluene. A Gaussian fit function is only required in the TiBuO-TiEtO mixtures for obvious reasons. Evidently the difference between locally favoured structures and liquid-like structures in $\mathrm{TiBuO}$ is too subtle to enable their distinction. It could be speculated that, as 
$\mathrm{TiBuO}$ forms trimeric clusters, the collision-induced signal is more sensitive to intra-cluster rather than inter-cluster dynamics.

We have shown here that symmetry (even if sometimes imperfect) can be used to simplify depolarised Raman and OKE spectra to bring out the boson peak unobscured by other contributions in the spectrum. In the case of $\mathrm{TiBuO}$, no separate contributions from liquid-like and locally favoured structures were observed, possibly due to its trimeric cluster nature. This suggests that if similar glass-forming liquids consisting of monomeric symmetric molecules can be found, the boson peak will be more sensitive to inter-cluster dynamics and hence the difference between locally favoured vs. liquid like clusters. Thus, this opens the way to the investigation of the detailed changes in the boson-peak shape as a function of temperature, pressure, fragility, and other physicochemical parameters, greatly aiding the science of the glassy state.

\section{Methods}

Sample preparation. Liquids were purchased from SigmaAldrich and have a rated purity $\geq 98 \%$ with the exceptions of titanium tetraethoxide, which has a purity $\geq 80 \%$ (5\% contamination with titanium tetraisopropoxide resulting in $20 \%$ titanium triethoxide monoisopropoxide) and were used without further purification. Liquid samples were filtered with a PTFE filter (Millex) with $20 \mu \mathrm{m}$ pore size and degassed for I min in an ultrasound bath before measurements. Room-temperature samples were contained in a I mm thick rectangular quartz cuvette (Starna) and held in a temperature-controlled $( \pm 0.5 \mathrm{~K})$ aluminium block. Low-temperature measurements were performed using a liquid- $\mathrm{N}_{2}$ cryostat (Oxford Instruments, $\pm 0 . \mathrm{I} \mathrm{K}$ ) in a nitrogen environment to avoid water condensation.

OKE experimental details. The OKE data were recorded in a standard time-domain pump-probe configuration and Fourier transformed to obtain the frequency-domain reduced depolarised Raman spectrum as described previously. ${ }^{46}$ A laser oscillator (Coherent Micra) produced $\sim 10 \mathrm{~nJ}$ pulses at a repetition rate of $82 \mathrm{MHz}$ and with $800 \mathrm{~nm}$ nominal wavelength providing $20 \mathrm{fs}$ temporal pulse width in the sample, broadening to 25 fs when using a cryostat.

OKE data analysis. OKE spectra consist of a number of broad overlapping bands that are either overdamped or underdamped, which are analysed through curve fitting to the imaginary part of a number of analytical complex functions. The overdamped or diffusive modes (corresponding to orientational and translational diffusion) are typically fit to the imaginary part of the Havriliak-Negami function, which is defined in the frequency domain by

$$
H_{\alpha, \beta}(\omega)=\frac{1}{\left(1+(-i \omega \tau)^{\alpha}\right)^{\beta}}
$$

with $0<\alpha, \beta \leq \mathrm{I}$ and where $\omega$ is the angular frequency and $\tau$ is the relaxation time. For $\beta=\mathrm{I}$, it reduces to the Cole-Cole function; for $\alpha=$ I it reduces to the Cole-Davidson function; and for $\alpha=\beta=\mathrm{I}$ it reduces to the Debye function. Near $\omega=0$, this function behaves as $\omega^{\alpha-\beta}$. Thus, it is clear that the Debye, Cole-Cole, Cole-Davidson and Havriliak-Negami functions will not show a boson peak for any setting of their parameters as they are all proportional to $\omega$ near the frequency origin.
More specifically, at the lowest frequencies, one typically finds processes associated with diffusive orientational relaxation of the molecules. These have been fit with the Debye function. Diffusive translational relaxation has been fit with the Cole-Cole function. However, it would be unphysical if diffusive processes were to be faster than the corresponding underdamped motion. So, diffusive orientational relaxation ought to be slower than the librations and diffusive translational relaxation ought to be slower than intermolecular cage-rattling modes. This inertial effect is accommodated using a rise function in the time-domain as described previously. ${ }^{44}$ Thus, translational relaxation is fit with an "inertial" Cole-Cole function defined by

$$
S_{i C C}(\omega)=\operatorname{Im}\left[H_{\alpha, 1}(\omega)-H_{\alpha, 1}\left(\omega+i \gamma_{\text {rise }}\right)\right],
$$

where $\gamma_{\text {rise }}$ is the frequency of the intermolecular mode. Similarly, orientational relaxation (when present as in toluene) is fit with an "inertial" Debye function defined by

$$
S_{i D}(\omega)=\operatorname{Im}\left[H_{1,1}(\omega)-H_{1,1}\left(\omega+i \gamma_{l i b}\right)\right]
$$

where $\gamma_{l i b}$ is the librational frequency.

In the terahertz range, one finds bands from modes that are not diffusive but critically damped or underdamped. These originate in librations, vibrations, and phonon-like modes. These have been fitted using the Brownian oscillator function $^{46,61}$

$$
S_{B O}(\omega)=\operatorname{Im} \frac{\omega_{0}^{2}}{\omega_{0}^{2}-\omega^{2}-i \gamma \omega},
$$

where $\omega_{0}$ is the undamped oscillator angular frequency and $\gamma$ is the damping rate. Sometimes, it is required to take into account inhomogeneous broadening and the band is fitted using the antisymmetrised Gaussian function defined by

$$
S_{G}(\omega)=\frac{1}{\sigma \sqrt{2 \pi}}\left[e^{-\frac{\left(\omega-\omega_{0}\right)^{2}}{2 \sigma^{2}}}-\mathrm{e}^{-\frac{\left(\omega+\omega_{0}\right)^{2}}{2 \sigma^{2}}}\right]
$$

where $\sigma$ is a width parameter.

In the overdamped limit, both the Brownian oscillator and antisymmetrised Gaussian functions are proportional to $\omega$ near the frequency origin and would therefore not be able to display a peak in $g(\omega) / \omega^{2}$, where $g(\omega)$ is the vibrational density of states. However, when underdamped, both will display a peak in $g(\omega) / \omega^{2}$.

Differential scanning calorimetry. Differential scanning calorimetry measurements were carried out with a TA Instruments DSC 2500 differential scanning calorimeter equipped with a Quench Cooling Accessory. The samples were cooled from $40^{\circ} \mathrm{C}$ with liquid nitrogen in circa I6 minutes to a temperature of $-\mathrm{I} 65^{\circ} \mathrm{C}$.

FTIR. Fourier-transform infrared (FTIR) spectroscopy measurements used a Bruker Vertex 70 spectrometer purged with dry air. liquid samples were sandwiched between two $\mathrm{ZnSe}$ windows. Sub-ambient FTIR experiments were performed using the same liquid- $\mathrm{N}_{2}$ cryostat with $\mathrm{ZnSe}$ windows. 
Viscometry. Variable temperature viscosity measurements were performed on an Anton Paar MCR 30I rheometer using a $50 \mathrm{~mm}$ cone (cone angle $=\mathrm{I}^{\circ}$, gap $=0 . \mathrm{IOI} \mathrm{mm}$ ) at a fixed shear rate of IO rad/s. The temperature was lowered from $75^{\circ} \mathrm{C}$ to $40^{\circ} \mathrm{C}$ at a rate of $\mathrm{I}^{\circ} \mathrm{C} / \mathrm{min}$ and the viscosity recorded every minute. Measurements were performed under an atmosphere of nitrogen to prevent oxidation of the sample using a custommade $3 \mathrm{D}$ printed plate hood. A cylinder of nitrogen was attached to an inlet on the hood to allow for a stream of nitrogen to pass over the measurement system and sample throughout the measurement. To help the temperature reach and maintain $-40^{\circ} \mathrm{C}$, the nitrogen line was passed through liquid nitrogen before it reached the hood on the rheometer. Measurements were performed in triplicate.

Conformer stability and normal mode calculations. All calculations were performed using the ORCA quantum chemistry program. ${ }^{62}$ A preliminary conformational search to find lowenergy TiO skeletons for titanium alkoxides trimers was carried out using the semiempirical GFN2-xTB method. ${ }^{63}$ Geometry optimisations and vibrational spectral calculations were carried out with the PBE exchange-correlation functional ${ }^{64}$ with the Grimme dispersion correction ${ }^{66}$ combined with def2SVP basis set. ${ }^{67} \mathrm{PBE}$ is a nonempirical GGE functional applicable to a broad range of systems from simple organic molecules to inorganic solids and metals, while the def2-SVP basis set is known to provide a good trade-off between accuracy and cost. To estimate errors arising due to basis set incompleteness, calculations with triple-zeta (def2-TZVP, def2-TZVPP) basis sets were also performed. To validate energy ordering of different titanium alkoxide trimers, PBEo-D3(BJ)/def2TZVPP calculations in hexane were conducted. Solvent effects were accounted for by using the polarisable continuum model $^{68}(\mathrm{CPCM})$ with the dielectric constant $\varepsilon_{\text {static }}=\mathrm{I} .89$ (hexane). For all DFT calculations, the RI approximation was employed.

It is known that titanium ethoxide and butoxide form trimers in neat liquid and solution (except at high dilution). ${ }^{54-56}$ In order to find low-energy trimers, various $\mathrm{Ti}_{3} \mathrm{O}_{\mathrm{I} 2}$ cores were cut from available crystal structures for rutile and anatase $\mathrm{TiO}_{2}$ polymorphs, saturated with hydrogens, and optimised with the GFN2-xTB method, resulting in four different conformers. Then, the hydrogens were substituted by methyls, ethyls, or butyls, and DFT optimisations were performed. Also, $\mathrm{Ti}_{3} \mathrm{O}_{\text {I2 }}$ cores were extracted from $\mathrm{Ti}(\mathrm{OR})_{4}$ crystal structures from the Cambridge Structural Database. Their optimisation as $\mathrm{Ti}_{3}(\mathrm{OH})_{12}$ yielded one more stable conformer, thus leading to five different minima on the PES (see Supplementary Note 4 and Supplementary Figure Io). All geometry optimisations were performed without symmetry constraints. $\mathrm{Ti}_{3} \mathrm{O}_{\mathrm{I} 2}$ cores were found to be close to $\mathrm{S}_{6}$ (I), $\mathrm{C}_{2}$ (III, IV) and $\mathrm{C}_{\mathrm{s}}$ (II, V) point groups. Harmonic analyses of these structures showed that all Hessian eigenvalues are positive. Structures I-V were compared to those proposed in the literature based on generic considerations ${ }^{56}$ or theoretical studies. ${ }^{69}$ Structures I and III correspond to I and II in Refs. ${ }^{55,69}$, structure IV is known from crystal trimers. However, it appears that structures II and V have not been considered previously. Structures III and IV proposed in Ref. ${ }^{56}$ are not stable and undergo rearrangement to one of the five stable conformers during geometry optimisation.

We did not aim to carry out a full conformational analysis including the preferred arrangement of alkyl chains in solution. Thus, the orientation of alkyl groups is arbitrary (and for butoxides the butyls have the zigzag conformation). Relative enthalpies and Gibbs energies were estimated within the rigid rotator-harmonic oscillator model.

IR and depolarised Raman spectra were obtained in the double harmonic approximation; the latter was converted to an OKE spectrum using equation S5. Spectra were broadened with Lorentzians with $\mathrm{I} 2 \mathrm{~cm}^{-1}$ half-width.

\section{Data availability}

The data that support the findings of this study are available in Enlighten: Research Data Repository (University of Glasgow) with the identifier: http://dx.doi.org/I0.5525/gla.researchdata.@@.

\section{References}

I. Angell, C. A., Ngai, K. L., McKenna, G. B., McMillan, P. F. \& Martin, S. W. Relaxation in glassforming liquids and amorphous solids. J. Appl. Phys. 88, 3II3-3I57 (2000).

2. Ediger, M. D. Spatially Heterogeneous Dynamics in Supercooled Liquids. Annu. Rev. Phys. Chem. 5I, 99-I28 (2000).

3. Martinez, L.-M. \& Angell, C. A. A thermodynamic connection to the fragility of glass-forming liquids. Nature 4IO, 663-667 (200I).

4. Debenedetti, P. G. \& Stillinger, F. H. Supercooled liquids and the glass transition. Nature 4I0, 259-267 (200I).

5. Dyre, J. C. Colloquium: The glass transition and elastic models of glass-forming liquids. Rev. Mod. Phys. 78, 953972 (2006).

6. Grigera, T. S., Martín-Mayor, V., Parisi, G. \& Verrocchio, P. Phonon interpretation of the 'boson peak' in supercooled liquids. Nature 422, 289-292 (2003).

7. Winterling, G. Very-low-frequency Raman scattering in vitreous silica. Phys. Rev. B I2, 2432-2440 (I975).

8. Schober, H. R. Vibrations and relaxations in a soft sphere glass: boson peak and structure factors. J. Phys. Condens. Matter I6, S2659-S2670 (2004).

9. Shintani, H. \& Tanaka, H. Universal link between the boson peak and transverse phonons in glass. Nat. Mater. 7 , 870-877 (2008).

IO. Schirmacher, W., Ruocco, G. \& Scopigno, T. Acoustic Attenuation in Glasses and its Relation with the Boson Peak. Phys. Rev. Lett. 98, 02550I (2007).

II. Sokolov, A. P. Vibrations at the boson peak: random- and coherent-phase contributions. J. Phys. Condens. Matter II, A2I3-A2I8 (I999).

I2. Schirmacher, W. Thermal conductivity of glassy materials and the "boson peak". EPL Europhys. Lett. 73, 892 (2006).

I3. Chumakov, A. I. et al. Equivalence of the Boson Peak in Glasses to the Transverse Acoustic van Hove Singularity in Crystals. Phys. Rev. Lett. I06, 22550I (20II).

I4. Taraskin, S. N., Loh, Y. L., Natarajan, G. \& Elliott, S. R. Origin of the Boson Peak in Systems with Lattice Disorder. Phys. Rev. Lett. 86, I255-I258 (200I).

I5. Schober, H. R., Buchenau, U. \& Gurevich, V. L. Pressure dependence of the boson peak in glasses: Correlated and uncorrelated perturbations. Phys. Rev. B 89, oI4204 (20I4).

I6. Parshin, D. A., Schober, H. R. \& Gurevich, V. L. Vibrational instability, two-level systems, and the boson peak in glasses. Phys. Rev. B 76, 064206 (2007).

I7. Gurevich, V. L., Parshin, D. A. \& Schober, H. R. Anharmonicity, vibrational instability, and the Boson peak in glasses. Phys. Rev. B 67, 094203 (2003). 
I8. Tanaka, H. Two-order-parameter model of the liquid-glass transition. III. Universal patterns of relaxations in glassforming liquids. J. Non-Cryst. Solids 35I, 3396-34I3 (2005).

I9. Farrell, A. J., González-Jiménez, M., Ramakrishnan, G. \& Wynne, K. Low-Frequency (Gigahertz to Terahertz) Depolarized Raman Scattering Off $n$-Alkanes, Cycloalkanes, and Six-Membered Rings: A Physical Interpretation. J. Phys. Chem. B I24, 76II-7624 (2020).

20.Lunkenheimer, P., Schneider, U., Brand, R. \& Loid, A. Glassy dynamics. Contemp. Phys. 4I, I5-36 (2000).

2I. Brodin, A., Börjesson, L., Engberg, D., Torell, L. M. \& Sokolov, A. P. Relaxational and vibrational dynamics in the glass-transition range of a strong glass former $\mathrm{B}_{2} \mathrm{O}_{3}$. Phys. Rev. B 53, II5II-II520 (I996).

22. Sokolov, A. P., Rössler, E., Kisliuk, A. \& Quitmann, D. Dynamics of strong and fragile glass formers: Differences and correlation with low-temperature properties. Phys. Rev. Lett. 7I, 2062-2065 (I993).

23. Surovtsev, N. V., Pugachev, A. M., Nenashev, B. G. \& Malinovsky, V. K. Low-frequency Raman scattering in $\mathrm{As}_{2} \mathrm{~S}_{3}$ glass former around the liquid-glass transition. J. Phys. Condens. Matter I5, 765I-7662 (2003).

24. Kojima, S. \& Novikov, V. N. Correlation of temperature dependence of quasielastic-light-scattering intensity and alpha-relaxation time. Phys. Rev. B 54, 222-227 (I996).

25. Baran, J., Davydova, N. A. \& Drozd, M. The role of nucleation in vitrification of supercooled liquids. J. Phys. Condens. Matter 22, I55I08 (20I0).

26. Bobovich, Y. S. Disordered Media and Materials: A Review of Recent Structural Data and Trends in Application of Raman Spectroscopy. Glass Phys. Chem. 29, 33I-344 (2003).

27. Wuttke, J., Petry, W., Coddens, G. \& Fujara, F. Fast dynamics of glass-forming glycerol. Phys. Rev. E 52, 4026-4034 (I995).

28. Wilding, M. C. et al. Structural properties of $\mathrm{Y}_{2} \mathrm{O}_{3}-\mathrm{Al}_{2} \mathrm{O}_{3}$ liquids and glasses: An overview. J. Non-Cryst. Solids 407, 228-234 (20I5).

29. Rufflé, B. et al. Comparative analysis of the fast dynamics in the supercooled nonfragile glass-forming liquid $\mathrm{Na}_{0.5} \mathrm{Li}_{0.5} \mathrm{PO}_{3}$ observed by coherent neutron scattering. Phys. Rev. B 56, II546-II552 (I997).

30. Yannopoulos, S. N., Kalampounias, A. G., Chrissanthopoulos, A. \& Papatheodorou, G. N. Temperature induced changes on the structure and the dynamics of the "tetrahedral" glasses and melts of $\mathrm{ZnCl}_{2}$ and $\mathrm{ZnBr}_{2}$. J. Chem. Phys. II8, 3I97-32I4 (2003).

3I. Tölle, A. Neutron scattering studies of the model glass former ortho -terphenyl. Rep. Prog. Phys. 64, I473-I532 (200I).

32. Hong, L. et al. Influence of Pressure on Quasielastic Scattering in Glasses: Relationship to the Boson Peak. Phys. Rev. Lett. I02, I45502 (2009).

33. Ngai, K. L., Sokolov, A. \& Steffen, W. Correlations between boson peak strength and characteristics of local segmental relaxation in polymers. J. Chem. Phys. Io7, 52685272 (1997).

34. Andrikopoulos, K. S., Kalampounias, A. G., Falagara, O. \& Yannopoulos, S. N. The glassy and supercooled state of elemental sulfur: Vibrational modes, structure metastability, and polymer content. J. Chem. Phys. I39, I245OI (20I3).
35. Yannopoulos, S. N. \& Andrikopoulos, K. S. Raman scattering study on structural and dynamical features of noncrystalline selenium. J. Chem. Phys. I2I, 4747-4758 (2004).

36. Caponi, S., Fontana, A., Rossi, F., Baldi, G. \& Fabiani, E. Effect of temperature on the vibrational density of states in vitreous $\mathrm{SiO}_{2}$ : A Raman study. Phys. Rev. B 76, o9220I (2007).

37. Yannopoulos, S. N., Andrikopoulos, K. S. \& Ruocco, G. On the analysis of the vibrational Boson peak and low-energy excitations in glasses. J. Non-Cryst. Solids 352, 454I-455I (2006).

38. Deschamps, T., Martinet, C., de Ligny, D., Bruneel, J. L. \& Champagnon, B. Low-frequency Raman scattering under high pressure in diamond anvil cell: Experimental protocol and application to $\mathrm{GeO}_{2}$ and $\mathrm{SiO}_{2}$ boson peaks. J. NonCryst. Solids 358, 3I56-3I60 (20I2).

39. Schroeder, J. et al. Raman scattering and Boson peaks in glasses: temperature and pressure effects. J. Non-Cryst. Solids 349, 88-97 (2004).

40.Zanatta, M. et al. Debye to non-Debye scaling of the Boson peak dynamics: Critical behavior and local disorder in vitreous germania. J. Chem. Phys. I35, I74506 (20II).

4I. Baldi, G. et al. Connection between Boson Peak and Elastic Properties in Silicate Glasses. Phys. Rev. Lett. I02, 195502 (2009).

42. Tanaka, H. Two-order-parameter model of the liquid-glass transition. II. Structural relaxation and dynamic heterogeneity. J. Non-Cryst. Solids 35I, 3385-3395 (2005).

43. Caporaletti, F. et al. A microscopic look at the Johari-Goldstein relaxation in a hydrogen-bonded glass-former. Sci. Rep. 9, I43I9 (20I9).

44. Reichenbach, J. et al. Phonon-like Hydrogen-Bond Modes in Protic Ionic Liquids. J. Am. Chem. Soc. 139, 7I60-7I63 (20I7).

45. González-Jiménez, M. et al. Observation of coherent delocalized phonon-like modes in DNA under physiological conditions. Nat. Commun. 7, II799 (20I6).

46. Turton, D. A. et al. Terahertz underdamped vibrational motion governs protein-ligand binding in solution. Nat. Commun. 5, 3999 (2014).

47. Tanaka, H. Bond orientational order in liquids: Towards a unified description of water-like anomalies, liquid-liquid transition, glass transition, and crystallization: Bond orientational order in liquids. Eur. Phys. J. E 35, II3 (20I2).

48. Scott, D. W. et al. Toluene: Thermodynamics Properties, Molecular Vibrations, and Internal Rotation. J. Phys. Chem. 66, 9II-9I4 (I962).

49. Yamamuro, O. et al. Calorimetric Study of Glassy and Liquid Toluene and Ethylbenzene: Thermodynamic Approach to Spatial Heterogeneity in Glass-Forming Molecular Liquids. J. Phys. Chem. B I02, I605-I609 (1998).

50. Wiedersich, J., Surovtsev, N. V. \& Rössler, E. A comprehensive light scattering study of the glass former toluene. J. Chem. Phys. II3, II43-II53 (2000).

5I. Wuttke, J. et al. Mode-coupling crossover in viscous toluene revealed by neutron and light scattering. Eur. Phys. J. $B$ - Condens. Matter Complex Syst. I, I69-I72 (I998).

52. Chumakov, A. I. et al. Collective Nature of the Boson Peak and Universal Transboson Dynamics of Glasses. Phys. Rev. Lett. 92, 245508 (2004).

53. Ibers, J. A. Crystal and Molecular Structure of Titanium (IV) Ethoxide. Nature I97, 686-687 (I963). 
54. Caughlan, C. N., Smith, H. S., Katz, W., Hodgson, Wm. \& Crowe, R. W. Organic Compounds of Titanium. II. Association of Organic Titanates in Benzene Solution. J. Am. Chem. Soc. 73, 5652-5654 (I95I).

55. Babonneau, F. et al. XANES and EXAFS study of titanium alkoxides. Inorg. Chem. 27, 3I66-3I72 (I988).

56. R.L. Martin \& G. Winter. Structure of the Trinuclear Titanium (IV) Alkoxides. Nature I88, 3I3-3I5 (I960).

57. C. Bradley, D. \& E. Holloway, C. Nuclear magnetic resonance and cryoscopic studies on some alkoxides of titanium, zirconium, and hafnium. J. Chem. Soc. A I3I6-I3I9 (I968) doi:Io.I039/Ji968000I3I6.

58. Turton, D. A. \& Wynne, K. Universal nonexponential relaxation: Complex dynamics in simple liquids. J. Chem. Phys. I3I, 20IIOI (2009).

59. Sette, F., Krisch, M. H., Masciovecchio, C., Ruocco, G. \& Monaco, G. Dynamics of Glasses and Glass-Forming Liquids Studied by Inelastic X-ray Scattering. Science $\mathbf{2 8 0}$, I550-I555 (I998).

6o. Bender, J. S., Zhi, M. \& Cicerone, M. T. The polarizability response of a glass-forming liquid reveals intrabasin motion and interbasin transitions on a potential energy landscape. Soft Matter I6, 5588-5598 (2020).

6I. Cho, M., Du, M., Scherer, N. F., Fleming, G. R. \& Mukamel, S. Off-resonant transient birefringence in liquids. J Chem Phys 99, I9 (I993).

62.Neese, F. The ORCA program system. WIREs Comput. Mol. Sci. 2, 73-78 (20I2).

63. Bannwarth, C., Ehlert, S. \& Grimme, S. GFN2-xTB-An Accurate and Broadly Parametrized Self-Consistent TightBinding Quantum Chemical Method with Multipole Electrostatics and Density-Dependent Dispersion Contributions. J. Chem. Theory Comput. I5, I652-I67I (2019).

64.Perdew, J. P., Burke, K. \& Ernzerhof, M. Generalized Gradient Approximation Made Simple. Phys. Rev. Lett. 77, 3865-3868 (1996).

65. Yanai, T., Tew, D. P. \& Handy, N. C. A new hybrid exchange-correlation functional using the Coulomb-attenuating method (CAM-B3LYP). Chem. Phys. Lett. 393, 5I-57 (2004).

66. Grimme, S., Antony, J., Ehrlich, S. \& Krieg, H. A consistent and accurate $\mathrm{ab}$ initio parametrization of density functional dispersion correction (DFT-D) for the 94 elements H-Pu. $J$. Chem. Phys. 132, I54IO4 (20I0).

67. Weigend, F. \& Ahlrichs, R. Balanced basis sets of split valence, triple zeta valence and quadruple zeta valence quality for $\mathrm{H}$ to $\mathrm{Rn}$ : Design and assessment of accuracy. Phys. Chem. Chem. Phys. 7, 3297-3305 (2005).

68. Barone, V. \& Cossi, M. Quantum Calculation of Molecular Energies and Energy Gradients in Solution by a Conductor Solvent Model. J. Phys. Chem. A I02, I995-200I (I998).

69.Ignatyev, I. S., Montejo, M. \& López González, J. J. DFT predictions of vibrational spectra of titanium tetramethoxide oligomers and the structure of titanium tetraalkoxides in liquid and solid phases. Vib. Spectrosc. 5I, 2I8-225 (2009).

\section{Acknowledgments}

We thank the Engineering and Physical Sciences Research Council (EPSRC) for support through grants EP/Joo9733/I, EP/Ko34995/I, EP/N508792/I, and EP/Noo74I7/I. This work was part funded by Leverhulme Trust Research Project Grant RPG-20I8-350 and received funding from the European Research Council (ERC) under the European Union's Horizon 2020 research and innovation program (grant agreement No. 832703). We would also like to thank Dr. Emily Draper (University of Glasgow) for carrying out viscosity measurements.

\section{Author contributions}

All authors contributed to the study and manuscript. AJF, MGJ, DAT, and SG were responsible for OKE data collection and their analysis. AJF and SG investigated a range of symmetric-molecule liquids for suitability in this study and carried out FTIR measurements. NT and HMS were responsible for the computer simulations. BAR was responsible for the DSC experiments. AJF, NT, HMS, and KW carried out data analysis and manuscript preparation.

\section{Competing interests}

The authors declare no competing interests.

\section{Additional information}

Additional information is linked to the online version of the paper at www.nature.com/nature.

\section{Corresponding author}

Klaas Wynne - University of Glasgow, School of Chemistry, Glasgow GI2 8QQ, UK; email: klaas.wynne@glasgow.ac.uk 